\title{
Evidence-Based Care for People with Unhealthy Alcohol Use-Still Elusive
}

\author{
Marcus A. Bachhuber, MD, MSHP' and Katharine A. Bradley, MD, MPH $H^{2,3,4}$ \\ 'Division of General Internal Medicine, Albert Einstein College of Medicine/Montefiore Medical Center, Bronx, NY, USA; ${ }^{2}$ Group Health Research \\ Institute, Seattle, WA, USA; ${ }^{3}$ Departments of Medicine and Health Services, University of Washington, Seattle, WA, USA; ${ }^{4} \mathrm{HSR} \& D$ Center of \\ Innovation, VA Puget Sound, Seattle, WA, USA.
}

J Gen Intern Med 31(7):710-1

DOI: $10.1007 / \mathrm{s} 11606-016-3695-1$

(C) Society of General Internal Medicine 2016

I n 1982, the World Health Organization (WHO) embarked on a multi-year, multi-country Collaborative Project on Identification and Treatment of Persons with Harmful Alcohol Consumption. ${ }^{1}$ The project reflected a major shift in thinking at the time, and was followed in 1990 by a seminal Institute of Medicine report that came to the same conclusion. ${ }^{2}$ Recognizing the considerable morbidity and mortality caused by alcohol, the new vision was to reduce unhealthy drinking at the population level. Instead of only addressing alcohol use through specialty treatment facilities, the WHO Collaborative Project sought to develop tools to screen for unhealthy drinking, and brief interventions to reduce it, among general medical patients.

In the decades since, studies from the WHO Collaborative Project and other groups have proven the efficacy of what we now call Screening and Brief Intervention (SBI). At its core, SBI seeks to reduce the consequences of unhealthy drinking (e.g., injuries) and to prevent progression to more serious alcohol use disorders (AUDs). Unhealthy drinking patterns can be uncovered through universal screening with validated instruments. For those with unhealthy levels of drinking, screening can prompt a brief intervention, including patientcentered feedback linking patients' alcohol use to their health, and advice to cut back below recommended limits. ${ }^{3}$ When screening identifies people with AUDs or severe consequences from their drinking, experts have historically recommended that such patients be referred to specialty treatment, because SBI is unproven for those with moderate to severe AUD. ${ }^{4}$

Due to its efficacy, alcohol SBI in primary care settings is widely recommended. After a review of the evidence, the US Preventive Services Task Force (USPSTF) gave alcohol SBI in adult primary care patients a "B" recommendation ("The USPSTF recommends the service"). ${ }^{5}$ In a ranking of all USPSTF-recommended practices based on the preventable burden of disease and cost effectiveness, the National

Published online April 12, 2016
Commission on Prevention Priorities found alcohol SBI to be similar in priority to colorectal cancer screening, hypertension screening, and influenza and pneumococcal vaccination. ${ }^{6}$

Despite the recommendation, provision of alcohol SBI has historically been much lower than provision of other preventive services. ${ }^{6}$ The Affordable Care Act brought reimbursement changes aimed at increasing its delivery. It expanded coverage of USPSTF-recommended preventive services without cost sharing by many private insurance plans as well as Medicaid expansion plans and Medicare. While the covered service is a minimum 15 to $30 \mathrm{~min} \mathrm{BI-impractical} \mathrm{for} \mathrm{most}$ primary care providers - many plans reimburse for SBI performed by co-located behavioral health staff (e.g., social workers, psychologists, and nurses).

After decades of research and policies aimed at integrating SBI into routine primary care, where are we today? In this issue of the Journal of General Internal Medicine, Glass et al. report the frequency of clinical assessment of alcohol use in US adults attending ambulatory health care. ${ }^{7}$ Among those with unhealthy drinking or AUDs, they also examine whether respondents received advice to cut back, were referred to specialty treatment, and used specialty treatment services.

Using the 2013 National Survey of Drug Use and Health, they found that nearly three-quarters $(71.1 \%)$ of adults attending at least one ambulatory care visit in the past year reported that a physician asked them how much they drink, how often they drink, or if they have had any problems from drinking. As this survey consists of an extensive computer-assisted interview to quantify substance use and substance use disorders, the authors were then able to look at the self-reported receipt of appropriate services among people with unhealthy alcohol use with or without AUD. Among those with unhealthy drinking, but not AUD, only about one in 20 of those assessed reported that their physician advised them to cut back. Among those with AUD who were assessed by a physician, a similar or even smaller fraction reported being offered information about treatment (depending on AUD severity).

Their analyses are rigorous, but, as the authors acknowledge, the data are subject to some of the typical limitations of such large surveys. For example, we do not specifically know why or how respondents' alcohol use was assessed. Some could have been assessed because they sought medical attention due to problems caused or exacerbated by alcohol use; 
however, the fact that $71 \%$ reported being assessed suggests those patients would have been a minority. More importantly, we also do not know if the physician actually uncovered or diagnosed the unhealthy drinking through the assessment conducted.

The good news is that the vast majority of Americans, although not everyone, are being asked by their health care providers about alcohol use. The bad news is that Glass and colleagues' study suggests that recent expansions to reimbursement have not led to widespread provision of BI or uptake of AUD treatment. Very few individuals who could benefit from BIs are receiving them. Furthermore, most patients with AUDs are not even being offered information on AUD treatment options.

How can we improve delivery of SBI? Several integrated health systems have shown that the combination of clinical decision support in electronic health records, performance measurement and feedback to providers can result in sustained implementation of SBI in primary care. ${ }^{8}$ Results suggest a team-based approach: with screening by staff who triage, assess vitals and room patients, and BIs offered by primary care providers. While short BIs appear to be as effective as longer ones -5 min was equivalent to 45 in a WHO study ${ }^{1}$ - the USPSTF review suggested that repeated BIs might be most effective. ${ }^{5}$ Therefore, modifying reimbursement to allow for truly brief interventions provided repeatedly over time might provide the most benefit to patients.

What should be offered to patients with AUDs? A recent systematic review of SBI interventions (also led by Glass) did not find that they increased uptake of specialty AUD treatment. ${ }^{9}$ Even when the interventions included a dedicated referral to treatment component, there were no differences from controls in the proportion treated for AUD. This makes sense when considering that screening identifies patients who are, by definition, not seeking treatment. In the absence of other motivating interventions, a simple referral to treatment is unlikely to be successful in most cases. Ongoing research is addressing this critical issue.

Almost 35 years after the start of the WHO Collaborative Project, integration of evidence-based care for unhealthy drinking into primary care remains elusive. There is a consensus among many experts that new models of care for AUDs are needed. William Miller - the alcohol treatment expert who developed motivational interviewing along with Stephen Rollnick-has recently written an elegant narrative review summarizing and extending some of these approaches. ${ }^{10} \mathrm{He}$ alerts us to the importance of "empathic listening with acceptance and respect" and collaborative decision-making with patients. As Miller points out, we need to develop a continuum of care, integrated into primary care and other medical settings, that offers evidence-based prevention and treatment options including medications, brief counseling and motivational interviewing - all proven efficacious. We also need systems and policies that support this care with resources. But most important, we need to bring the same empathy and patient-centeredness used to manage other chronic medical conditions to the management of unhealthy alcohol use.

Acknowledgments: Dr. Bradley is supported by K24 AAO22128 from the National Institute on Alcohol Abuse and Alcoholism.

Corresponding Author: Marcus A. Bachhuber, MD, MSHP; Division of General Internal Medicine, Albert Einstein College of Medicine/ Montefiore Medical Center, 3300 Kossuth Ave, Bronx, NY 10467, USA (e-mail: mbachhub@montefiore.org).

Compliance with Ethical Standards:

Conflict of Interest: The authors have no potential conflict of interest to declare.

\section{REFERENCES}

1. WHO Brief Intervention Study Group. A cross-national trial of brief interventions with heavy drinkers. Am J Public Health. 1996:86:948-955.

2. Institute of Medicine. Broadening the Base of Treatment for Alcohol Problems. Washington, DC: National Academies Press; 1990.

3. National Institute on Alcohol Abuse and Alcoholism. Helping Patients Who Drink Too Much: A Clinician's Guide (Updated 2005 Edition). Washington, DC: National Institutes of Health, U.S. Department of Health and Human Services; 2005.

4. Saitz R. Alcohol screening and brief intervention in primary care: absence of evidence for efficacy in people with dependence or very heavy drinking. Drug Alcohol Rev. 2010;29:631-640.

5. Jonas DE, Garbutt JC, Amick HR, et al. Behavioral counseling after screening for alcohol misuse in primary care: a systematic review and meta-analysis for the U.S. Preventive Services Task Force. Ann Intern Med. 2012; 157:645-654.

6. Solberg LI, Maciosek MV, Edwards NM. Primary care intervention to reduce alcohol misuse ranking its health impact and cost effectiveness. Am J Prev Med. 2008;34:143-152.

7. Glass J, Bohnert K, Brown R. Alcohol screening and intervention among United States adults who attend ambulatory healthcare. J Gen Intern Med. 2016. doi:10.1007/s11606-016-3614-5.

8. Mertens JR, Chi FW, Weisner CM, et al. Physician versus non-physician delivery of alcohol screening, brief intervention and referral to treatment in adult primary care: the ADVISe cluster randomized controlled implementation trial. Addict Sci Clin Pract. 2015;10:26.

9. Glass JE, Hamilton AM, Powell BJ, Perron BE, Brown RT, Ilgen MA. Specialty substance use disorder services following brief alcohol intervention: a meta-analysis of randomized controlled trials. Addiction. 2015;110:1404-1415

10. Miller WR. Sacred cows and greener pastures: reflections from 40 years in addiction research. Alcohol Treat Q. 2016;34:92-115. 